\title{
Sider, the inheritance of intrinsicality, and theories of composition
}

\author{
Cody Gilmore
}

Published online: 14 July 2009

(C) The Author(s) 2009. This article is published with open access at Springerlink.com

\begin{abstract}
I defend coincidentalism (the view that some pluralities have more than one mereological fusion) and restricted composition (the view that some pluralities lack mereological fusions) against recent arguments due to Theodore Sider.
\end{abstract}

Keywords Sider · Intrinsic · Intrinsicality · Parts · Parthood · Composition · Universalism · Objects · Coinciding objects · Mereology · Metaphysics · Ontology $\cdot$ Material objects

\section{Introduction}

In a recent paper, Theodore Sider has proposed new arguments for a package of controversial views about parthood, including the following:

Unrestricted composition: Any Xs have a mereological sum

Uniqueness of composition: No Xs have more than one mereological sum (2007, p. 70$)^{1}$

Unrestricted composition (UC) says that any things, no matter how scattered and unrelated they might be, compose some further thing. There is, on this view, something that is composed of the moon and the Eiffel Tower. The Uniqueness of Composition (Uniqueness) says that it never happens that some things compose two or more further things. Uniqueness tells us that if some particles compose both a

\footnotetext{
1 Sider adopts the following definitions (2007, p. 52), stated a bit differently: $y$ is a mereological sum/ fusion of the Xs $=$ df. the Xs compose $\mathrm{y}=\mathrm{df}$. (i) each of the Xs is a part of $\mathrm{y}$, and (ii) each part of $\mathrm{y}$ overlaps (shares a part with) at least one of the Xs.
}

C. Gilmore $(\bowtie)$

University of California, Davis, Davis, CA, USA

e-mail: gilmore@ucdavis.edu 
statue-shaped lump of clay, Lumpl, and also a clay statue, Goliath, then Goliath = Lumpl, despite any apparent differences between them.

Sider's arguments for UC and Uniqueness are quite different from anything previously on the metaphysical landscape, and in my view they have a lot of initial appeal. But, as I will argue here, a core segment of their target audience has a surprising and potentially viable way to resist them.

\section{Sider's arguments}

The arguments have an inference-to-the-best-explanation structure familiar from the natural sciences. Certain propositions (the data) are taken to support certain other propositions (the predictions) indirectly, via an intermediary theory. The theory is said to do two things: (i) provide the best explanation of the data, and (ii) entail or otherwise 'yield' the predictions. When all goes well, the theory inherits justification from the data, and the predictions inherit justification from the theory. ${ }^{2}$

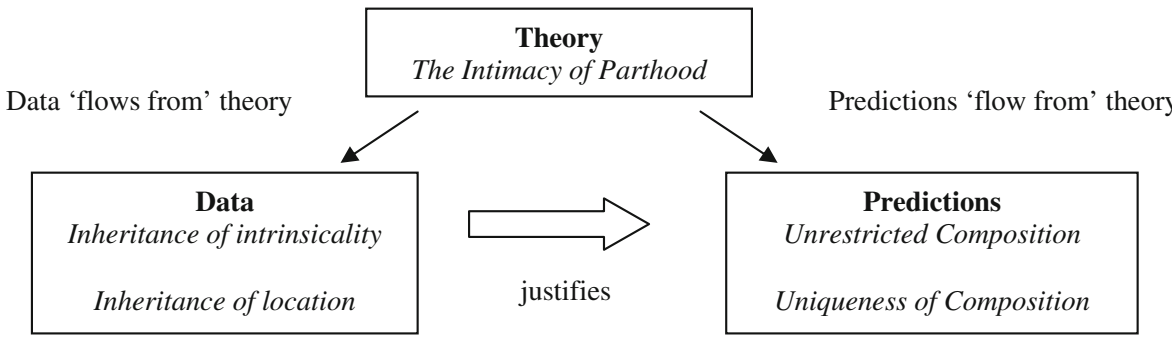

In the present case, the role of the predictions is played by Uniqueness and UC, and the role of the data is played by a pair of 'Inheritance' principles that Sider takes to be relatively uncontroversial:

Inheritance of Intrinsicality: If property $\mathrm{P}$ is intrinsic, then the property having a part that has $P$ is also intrinsic.

Inheritance of Location: If $\mathrm{x}$ is part of $\mathrm{y}$, then $\mathrm{y}$ is located wherever $\mathrm{x}$ is located (2007, p. 70).

Finally, the role of the theory is played by a rough and intuitive 'picture' of the part-whole relation:

The Intimacy of Parthood: The part-whole relationship is especially close: in some loose sense, a whole 'just is' or 'is nothing over and above' its parts.

To get a feel for Intimacy, it may help to contrast it with a stronger and more precise view that Sider rejects, namely:

Strong Composition as Identity: For any Xs and any y, if y is composed of the Xs, then $\mathrm{y}=$ the Xs (2007, p. 55).

\footnotetext{
2 The diagram is adapted from Sider (2007, p. 76).
} 
Suppose that an object-Michigan, say-is composed of two smaller objects, the Upper Peninsula and the Lower Peninsula. ${ }^{3}$ As applied to this case, Strong Composition as Identity (SCI) entails that Michigan is quite literally numerically identical to the Upper Peninsula and the Lower Peninsula. SCI does not entail that Michigan is identical to the peninsulas 'individually': it does not entail that Michigan $=$ the Upper Peninsula or that Michigan $=$ the Lower Peninsula. Rather it entails that Michigan is identical to the peninsulas 'collectively'. On this view, 'is identical to' is an irreducibly plural predicate, like 'surrounds'. (To borrow an example from Sider, "Tom, Dick, and Harry surrounded John" can be true even when "Tom surrounded John", "Dick surrounded John", and "Harry surrounded John" are all false.)

On its face, SCI is bizarre. How could Michigan be identical to the peninsulas, given that Michigan is one thing, whereas the peninsulas are two things? Sider is unwilling to reject SCI on the basis of initial worries such as this. (I assume that he is willing to say that, despite appearances, being one thing is compatible with being two things.) But he does reject SCI in the end, on the basis of theoretical considerations that we don't need to discuss here.

What is important for our purposes is just to see how SCI differs from Intimacy. SCI is a precisely stated but very radical thesis about composition and identity. Intimacy is less radical (in part because it is consistent with the standard view that the identity predicate is not irreducibly plural) and much less precise. It does not say that a composite object is strictly identical to the things that compose it, but merely that the object stands in an especially close relationship with those things: in some unspecified sense, it 'is nothing over and above' them. As a heuristic, we can think of Intimacy as the view that we get when we try to treat the relation between a composite object and the things that compose it as being as identity-like as possible, short of embracing full-blown SCI.

With the relevant views on the table, two questions arise right away.

(1) How is the theory supposed to explain the data? That is, how is the Intimacy of Parthood supposed to explain the Inheritance of Intrinsicality and Location? Briefly, Sider's line of thought is this. The Inheritance principles tell us that "the part-its intrinsic nature and location-is reflected in the whole. The part shines through" (2007, p. 70). Why does a thing's nature reflect the natures of its parts in this way? Because - as the Intimacy of Parthood states - the thing is nothing over and above those very parts; it 'just is' those parts! Now, this is not exactly a rigorous proof. Sider of course recognizes this, but he takes it to have some force anyway, and he claims that we cannot expect to do much better, given the nature of the territory: Intimacy is, after all, just a rough picture.

(2) Why should we think that Uniqueness and UC 'flow' from Intimacy? There are really two questions here, and Sider addresses them separately. First, why should the friend of Intimacy feel pressure to accept UC?

First, the intuitive picture of the intimacy of parthood demands that we adhere as closely as possible to strong composition as identity, and so requires

\footnotetext{
${ }^{3}$ We can ignore the many islands in the Great Lakes that are parts of the state but disjoint from both peninsulas.
} 
acceptance of unrestricted composition (though as we saw in Sect. 3.2, the relation between strong composition as identity and unrestricted composition is not straightforward). Second, consider the following line of thought, which is compelling though hard to evaluate. Unless composition is unrestricted, it is in general an open question whether some Xs compose something. But then in cases where some Xs do compose something, the composed object seems to be something 'over and above' the Xs. For it is an open question whether it exists, given that the Xs do, whereas it is not an open question whether the Xs exist, given that the Xs do. Finally, opposition to unrestricted compositiondistrust of scattered objects and the like-is undermined by the idea that the whole is 'nothing over and above the parts'. (2007, pp. 72-73)

Second, why can't the friend of Intimacy reject Uniqueness and say that in some cases, two different objects are composed of the very same things?

If some Xs compose two things, then wholes could not be "nothing over and above their parts," for how could distinct things each be nothing over and above the same parts? (2007, p. 70)

I will say no more about Sider's argument from Intimacy to UC. He takes it to be 'regrettably shaky' (2007, p. 72), but for the sake of argument I'll assume it goes through. In the next section I'll briefly comment on the case from Intimacy to Uniqueness, about which Sider does not express any doubts.

My main ambition, though, will be to challenge some of the data on which Sider's argument operates. Sider takes it that one will find both Inheritance principles to be plausible regardless of one's views on the controversies surrounding Uniqueness and UC (2007, p. 74), and on first blush this seems appropriate. In particular, it is natural to think that the Inheritance of Intrinsicality is neutral with respect to the debate between unrestricted composition and its rivals (compositional nihilism ${ }^{4}$ and various 'moderate' theories of composition).

I argue, however, that a core segment of Sider's target audience has independent reason for rejecting or at least being agnostic about the Inheritance of Intrinsicality. This segment includes some philosophers who accept, or at least take seriously, a theory of composition that embraces most of the composite objects of our commonsense ontology (organisms, artifacts, etc.) but that stops short of unrestricted composition. When such a theory is combined with a 'microphysical supervenience' principle that some have found appealing, the Inheritance of Intrinsicality becomes vulnerable to a surprising counterexample.

It turns out, then, that whether or not one should find the Inheritance of Intrinsicality plausible will depend upon one's prior inclinations regarding (i) theories of composition and (ii) the relevant sort of microphysical supervenience; the Inheritance of Intrinsicality is not neutral with respect to those views. I take this result to be interesting in its own right, even apart from any destructive effect that it might have upon Sider's arguments for Uniqueness and UC.

\footnotetext{
${ }^{4}$ Compositional nihilism is the view that there are no composite objects. An object is composite iff it has a proper part (a part with which it is not identical). An object is simple iff it is not composite.
} 


\section{Does intimacy require uniqueness?}

In defense of the claim that Uniqueness 'flows' from Intimacy, Sider asks, "how could distinct things each be nothing over and above the same parts?" (2007, p. 70) The short answer is: in whatever way distinct pluralities (e.g., the rows and the columns on a chessboard) could each be nothing over and above the same parts (the squares), perhaps by being nothing over and above each other.

Now for a longer answer. Sider's route from Intimacy to Uniqueness runs through the following principle:

P1 For any Xs, any $\mathrm{y}$, and any $\mathrm{z}$, if $\mathrm{y}$ is nothing over and above the $\mathrm{Xs}$, and $\mathrm{z}$ is nothing over and above the Xs, then $\mathrm{y}=\mathrm{z}$.

We can think of P1 as the uniqueness of nothing-over-and-above: it says that it never happens that two distinct objects are each nothing over and above the same plurality of things. It can be motivated by appeal to the thought that being nothing over and above should turn out to be as identity-like like as possible. After all, assuming that we allow ' $=$ ' to be flanked by 'mixed' pairs consisting of one singular term and one plural term, presumably everyone will grant:

$\mathrm{P} 1=$ For any Xs, any $\mathrm{y}$, and any $\mathrm{z}$, if $\mathrm{y}=$ the Xs, and $\mathrm{z}=$ the Xs, then $\mathrm{y}=\mathrm{z}^{5}$

So if P1 were false, this would be a respect in which being nothing over and above was unlike identity.

Despite this, I think that P1 is vulnerable. Now one might be unwilling to assent to P1 simply because one finds the predicate 'is nothing over and above' unintelligible. I have some sympathy for this reaction, but it would be nice to do better. So let's work under the assumption that 'is nothing over and above' is a wellunderstood primitive.

Here is a different concern. It seems to me that P1 is plausible only if it's backed by a more general principle, namely:

P1+ For any Xs, any Ys, and any Zs, if [the Ys are nothing over and above the Xs, the Zs are nothing over and above the Xs, and there are exactly as many of the $\mathrm{Ys}$ as there are of the $\mathrm{Zs}^{6}$, then the $\mathrm{Ys}=$ the $\mathrm{Zs}$.

Like P1, P1+ is motivated by the idea that we should make being nothing over and above as identity-like as possible. P1+ is more general, however, since it applies not just to single individuals but to pluralities as well; it says that it never happens that two distinct (but equinumerous) pluralities are each nothing over and the same plurality. So just as P1 can be thought of as the uniqueness of nothingover-and-above for individual things, $\mathrm{P} 1+$ can be thought of as the uniqueness of

\footnotetext{
${ }^{5}$ Even those who deny SCI can hold that some claims of the form ' $\mathrm{a}=$ the Bs' are true. For a plural term such as 'the Bs' can refer to some things of which there is only one. In that case, the singular referring term ' $a$ ' and plural referring term 'the Bs' might both refer to the very same entity, thus making ' $a=$ the Bs' true.

${ }^{6}$ Without this last clause the principle would be vulnerable to counterexamples such as the following: my cells are nothing over and above my atoms, and my molecules are nothing over and above my atoms, but (since I have more molecules than cells) my cells are not identical to my molecules.
} 
nothing-over-and-above for pluralities. I think we should expect $\mathrm{P} 1+$ to be true if $\mathrm{P} 1$ is; surely the latter is most appealing when it is seen as a special case of the former.

But as the 'short answer' hinted earlier, P1+ is in trouble. I assume that Intimacy-lovers will say that

(i) the rows in my chessboard are nothing over and above the squares in my chessboard,

(ii) the columns in my chessboard are nothing over and above the squares in my chessboard, and

(iii) there are exactly as many rows in my chessboard as there are columns in my chessboard. (There are eight of each.)

Together with $\mathrm{P} 1+$, these entail

(iv) the rows in my chessboard = the columns in my chessboard.

But (iv) should be rejected by all but friends of SCI. For the rows on my chessboard run horizontally, whereas the columns do not; they run vertically. So $\mathrm{P} 1+$ is too strong for Intimacy-lovers who reject SCI.

I think this reflects badly on P1. The Intimacy-lover is free to endorse the nonidentity of pluralities (e.g., the rows and the columns) each of which he takes to be nothing over the same things. So why not think that he's free to endorse the nonidentity of single individuals (e.g., Lumpl and Goliath) each of which he takes to be nothing over and above the same things? To reject P1+ (the uniqueness of nothingover-and-above for pluralities) but then stridently insist on P1 (the uniqueness of nothing-over-and-above for individuals) strikes me as being rather ad hoc.

This verdict is reinforced when we notice that there are appealing substitutes for these principles close at hand. The Intimacy-lover can account for the close connection between pluralities (or single things) that are nothing over and above the same things without holding that those pluralities (or single things) are identical. He can hold that they are nothing over and above each other. That is, he can endorse

P1* For any Xs, any Ys, and any Zs, if the Ys are nothing over and above the Xs, and the Zs are nothing over and above the Xs, then the Ys are nothing over and above the Zs.

The Intimacy-lover who accepts $\mathrm{P} 1 *$ will say that while the rows and the columns may be non-identical, the rows are nothing over and above the columns, and vice versa. Likewise for Lumpl and Goliath: though they may be non-identical, they are nothing over and above each other.

\section{The inheritance of intrinsicality}

The previous section shows that Sider's argument from Intimacy to Uniqueness can be resisted. The remainder of the paper focuses on a principle that Sider appeals to in support of Intimacy, the Inheritance of Intrinsicality: if a property P is intrinsic, 
then the property having a part that has $P$ is also intrinsic. It will be useful, therefore, to begin with a few informal remarks about the notion of intrinsicality.

To say that a property $\mathrm{P}$ is intrinsic is, very loosely, to say that whether or not a given object has $\mathrm{P}$ depends only on what the object is like in itself and is independent of how the object is related to things separate from itself. Put in different terms, intrinsic properties are the ones that cannot differ between intrinsic duplicates. Shapes and rest masses are typically regarded as intrinsic; properties such as being two miles from a lake are typically regarded as extrinsic (i.e., not intrinsic).

According to the Inheritance of Intrinsicality, if the property roundness is intrinsic, then so is the property having a part that has roundness. This is appealing: on the assumption that roundness is intrinsic, it would seem that if I have a round part, so must any intrinsic duplicate of me.

To get a better feel for the principle, it may help to contrast it with a pair of much stronger and less plausible variants. First, consider:

V1 For any property $\mathrm{P}$ whatever, intrinsic or extrinsic, the property having a part that has $P$ is intrinsic.

This is clearly false. Let $\mathrm{P}$ be the extrinsic property being in California. Then the hydrogen atoms in California have the property having a part that has $P$, but presumably there are intrinsic duplicates of them elsewhere that lack that property. Second, consider:

V2 For any intrinsic property $\mathrm{P}$ and any dyadic relation $R$ whatever, the property bearing $R$ to something that has $P$ is intrinsic.

This is no better. Even if roundness is intrinsic, the property bearing the being two feet away from relation to something that has roundness (roughly, being two feet away from a round thing) is not. So if either (i) the property $\mathrm{P}$ is itself extrinsic or (ii) $\mathrm{R}$ is some relation other than having as a part, then there is clearly no guarantee that the property bearing $R$ to something that has $P$ will be intrinsic. But when $\mathrm{P}$ is intrinsic and $\mathrm{R}$ is having as a part, it may initially seem that bearing $R$ to something that has $P$ must be intrinsic too.

In the next section, however, I will argue that in the presence of a pair of apparently respectable views about intrinsicality and composition, the Inheritance of Intrinsicality is vulnerable to a counterexample.

\section{The counterexample}

Here is a first pass; refinements will be made in response to objections. Suppose that we are attracted to the following theory of composition ${ }^{7}$ :

VIPA+ Some things, the Xs, compose something if and only if either:

\footnotetext{
${ }^{7}$ Markosian (2008) abbreviates van Inwagen's Proposed Answer (according to which some things compose something iff either there is just one of them or they are arranged living-organism-wise) as VIPA. Hence the name VIPA+.
} 
1. there is only one of the Xs, or

2. the Xs are arranged living-organism-wise (where this is a purely intrinsic matter), or

3. the Xs are arranged artifact-wise (where this is at least partly an extrinsic, historical matter that concerns the relation between the Xs' present internal arrangement and the intentions and activities of some sentient designer(s)).

Nothing will turn on accepting this answer in particular. As I explain in my reply to Objection 2, the relevant style of counterexample can be generated in the presence of a very wide range of moderate theories of composition. I introduce VIPA + merely to give us a clear and definite representative to work with initially. There are two points to note about this theory.

(1) To fix ideas, I will help myself to Lewis's notion of perfectly natural properties and relations. ${ }^{8}$ Whether or not some simple particles are arranged livingorganism-wise seems to supervene on their perfectly natural properties (perhaps masses, charges, spins) and on the perfectly natural relations (spatiotemporal and, perhaps, causal relations) that hold among the simples in question, independently of how those simples are related to other things.

(2) However, whether or not some simple particles are arranged artifact-wise is clearly an extrinsic matter, not entirely fixed by the perfectly natural properties of the simples and the perfectly natural relations that hold between them. It also depends upon their history, on how they came to be arranged as they are. Suppose that I have some particles, the Xs, arranged chair-wise in one corner of my office and some duplicate particles, the Ys, in exactly the same internal arrangement, located in another corner of my office. If the Xs came to be arranged in this way as a result of the right sort of intentional activity, then VIPA+ says that they compose something (a chair, presumably). It also says that if the Ys came to be arranged in that same way just by chance, then they don't compose anything, since in that case there are more than one of them and they are arranged neither living-organism-wise nor artifact-wise.

With these points in mind, consider two living human beings, Xavier and Yorick, both composed of simples. The simples that compose Xavier are the Xs, and those that compose Yorick are the Ys. Intuitively, the Xs match the Ys with respect to their intrinsic properties and internal arrangement. We can make this talk of matching more precise by saying that there is a one-to-one correspondence between the Xs and the Ys that preserves perfectly natural properties and relations-i.e., there is a bijective function $\mathrm{f}$ from the $\mathrm{Xs}$ to the Ys such that (i) for any $\mathrm{x}$ among the

\footnotetext{
${ }^{8}$ Roughly, these are the properties and relations that correspond to the primitive predicates in a complete physics and that ground objective resemblances. Following Lewis, I will assume that (a) all perfectly natural properties are intrinsic but that (b) many intrinsic properties (e.g., being either round or square) are not perfectly natural, and that (c) all intrinsic properties (hence all perfectly natural properties) and all perfectly natural relations are purely qualitative - roughly, not such as to 'constitutively involve' any specific concrete particulars. (Being two feet away from George W. Bush is not purely qualitative, whereas being two feet away from a man with gray hair is.) On perfectly natural properties, see Lewis (1986, pp. 60-69).
} 
$\mathrm{Xs}$ and any intrinsic property $\mathrm{P}, \mathrm{x}$ has $\mathrm{P}$ iff $\mathrm{f}(\mathrm{x})$ has $\mathrm{P}$ and (ii) for any ordered n-tuple $\left\langle\mathrm{x}_{1}, \ldots, \mathrm{x}_{\mathrm{n}}\right\rangle$ of the $\mathrm{Xs}$ and any perfectly natural, $\mathrm{n}$-adic relation $\mathrm{R}, \mathrm{x}_{1}, \ldots, \mathrm{x}_{\mathrm{n}}$ instantiate $\mathrm{R}$ (in that order) iff $\mathrm{f}\left(\mathrm{x}_{1}\right), \ldots, \mathrm{f}\left(\mathrm{x}_{\mathrm{n}}\right)$ instantiate $\mathrm{R}$ (in that order).

This makes it plausible that Xavier and Yorick are intrinsic duplicates, that they share all of their intrinsic properties. To see why, consider the following principle, which is a variant of a principle that Trenton Merricks calls the Principle of Microphysical Supervenience?

MS* Necessarily, for any objects $\mathrm{O}_{\mathrm{x}}$ and $\mathrm{O}_{\mathrm{y}}$, any simples, the $\mathrm{Xs}$, and any simples, the Ys, if (i) $\mathrm{O}_{\mathrm{x}}$ is composed of the Xs, (ii) $\mathrm{O}_{\mathrm{y}}$ is composed of the Ys, and (iii) there is a one-to-one correspondence between the $\mathrm{Xs}$ and the Ys that preserves perfectly natural properties and relations, then $\mathrm{O}_{\mathrm{x}}$ and $\mathrm{O}_{\mathrm{y}}$ are intrinsic duplicates: i.e., then for any intrinsic property $\mathrm{P}, \mathrm{O}_{\mathrm{x}}$ has $\mathrm{P}$ iff $\mathrm{O}_{\mathrm{y}}$ has P. ${ }^{10,11}$

MS* says that a sufficient condition for two things to be intrinsic duplicates is for them to be composed of matching simples in matching internal arrangements. MS* does not say that if the Xs and the Ys are matching simples in matching internal arrangements, then the Xs compose something iff the Ys compose something: it leaves open the possibility (endorsed by friends of VIPA+) that such pluralities of simples might still differ with respect to whether they compose anything. So MS* is neutral with respect to VIPA+ and similar 'externalist' theories of composition.

\footnotetext{
${ }^{9}$ The principle that Merricks discusses (and attacks) is: "Necessarily, if atoms $A_{1}$ through $A_{n}$ compose an object that exemplifies qualitative properties $Q_{1}$ through $Q_{n}$, then atoms like $A_{1}$ through $A_{n}$ (in all their respective intrinsic qualitative properties), related to one another by all the same restricted atom-to-atom relations as $A_{1}$ through $A_{n}$, compose an object that exemplifies $Q_{1}$ through $Q_{n}$ " (1998a, p. 59).

${ }^{10}$ This is a 'weak supervenience' claim: in possible worlds terminology, it says that any two objects within the same possible world that are alike in a certain way will be alike in another way as well. MS* is entailed by a corresponding 'strong supervenience' claim, $\mathrm{MS}^{*}+$, to the effect that for any possible worlds w1 and w2, and for any object $\mathrm{O} 1$ in w1 and any object $\mathrm{O} 2$ in $\mathrm{w} 2$, if $\mathrm{O} 1$ in $\mathrm{w} 1$ and $\mathrm{O} 2$ in w2 are alike with regard to the relevant base facts, then $\mathrm{O} 1$ in $\mathrm{w} 1$ and $\mathrm{O} 2$ in w2 will be alike with regard to the relevant supervening facts as well. I suspect that virtually everyone who finds MS* plausible will be attracted to the stronger principle too. Unsurprisingly, all the work done in this paper by MS* could also be done by MS*+. I use MS* merely because it's somewhat easier to state and because MS*+, though probably equally plausible, is not needed.

${ }^{11}$ MS* should be distinguished from a weaker principle, MS*-: Necessarily, for any objects $\mathrm{O}_{\mathrm{x}}$ and $\mathrm{O}_{\mathrm{y}}$, any simples, the Xs, and any simples, the Ys, and any intrinsic properties, the Ps, if (i) $\mathrm{O}_{\mathrm{x}}$ is composed of the $\mathrm{Xs}$, (ii) $\mathrm{O}_{\mathrm{y}}$ is composed of the $\mathrm{Ys}$, (iii) $\mathrm{O}_{\mathrm{x}}$ has each of the Ps, and (iii) there is a one-one correspondence between the Xs and the Ys that preserves perfectly natural properties and relations, then the Ys compose something (not necessarily $\mathrm{O}_{\mathrm{y}}$ ) that has each of the Ps. MS*-, but not MS*, leaves open the possibility that there are intrinsic properties that can differ between objects composed of the same simples. Perhaps some wish to claim that modal properties such as possibly existing while squashed or aesthetic properties such as being beautiful are intrinsic but can differ between a statue and a lump both composed of the same simples. MS*- allows this, while still guaranteeing that any matching simples in an internally matching arrangement, if they compose anything, must compose at least one thing that is an intrinsic duplicate of the statue and at least one thing that is an intrinsic duplicate of the lump. With one exception, the work that MS* does in this paper could also be done by MS*-. The exception concerns the proof that I give in the appendix. As I explain there, if MS* were replaced with MS*-, the proof would also need to invoke the mereological principle strong supplementation. (Like MS*, MS*- is a weak supervenience claim but can be modified in an obvious way to produce a corresponding strong supervenience claim.)
} 
Suppose now that Xavier has an artificial hand but that Yorick does not. Some of the Xs - those arranged right-hand-wise-were deliberately arranged in that way by a team of super-advanced biomedical engineers, who then seamlessly attached the artificial hand to Xavier's wrist. Then, according to VIPA+, those simples compose something-Xavier's right hand, presumably. Those of Yorick's constituent particles that are arranged right-hand-wise were not arranged in that way intentionally, however, so they aren't arranged artifact-wise. But nor are they by themselves arranged organism-wise. The result, then, is that they don't compose anything, according to VIPA+. So Yorick has no right hand (for if he did it would be composed of those simples). In fact, Yorick's only proper parts are his simple particles and perhaps his cells (if simples arranged cell-wise count as being arranged organism-wise). ${ }^{12}$ Xavier's parts, by contrast, include not just his simple particles (and cells, perhaps) but also a certain artifact — an artificial right hand.

Now consider some intrinsic property had by this hand but not by any of Xavier's other parts. Suppose that being hand-shaped is such a property, ${ }^{13}$ and consider the property of having a part that has that property: having a hand-shaped part. According to the Inheritance of Intrinsicality, since being hand-shaped is an intrinsic property, having a hand-shaped part must also be intrinsic.

But if VIPA+ is the correct theory of composition, and if MS* is true, then the case of Xavier and Yorick refutes the Inheritance of Intrinsicality. After all, MS* tells us that Yorick and Xavier are intrinsic duplicates, and VIPA+ tells us that only one of them-only Xavier-has the property having a hand-shaped part. ${ }^{14}$ No property with respect to which intrinsic duplicates can differ is intrinsic. Contrary to the Inheritance of Intrinsicality, then, having a hand-shaped part is not intrinsic, even though being hand-shaped is. Or so it can be argued by friends of VIPA+.

\section{Objections and replies}

Objection 1. MS*, which was used to argue that Xavier and Yorick are intrinsic duplicates, is refuted by the possibility of strongly emergent properties, where that term is defined as follows:

$\mathrm{F}$ is a strongly emergent property $=\mathrm{df}$. (i) $\mathrm{F}$ is a perfectly natural property, (ii) $\mathrm{F}$ can be exemplified by composite material objects, and (iii) $\mathrm{F}$ does not locally supervene on the perfectly natural properties and relations exemplified by only atomic material objects. (McDaniel 2008, p. 131)

\footnotetext{
12 Some friends of VIPA + might wish to leave open the possibility that there are objects composed of the Ys but not identical with Yorick. Such objects might also be among Yorick's proper parts.

13 The claim that shapes are intrinsic has been challenged (McDaniel 2003; Skow 2007). Perhaps those who doubt that shapes are intrinsic will be able to find some other property that is intrinsic even by their lights and that can play the same role in my argument as being hand-shaped.

${ }^{14}$ MS*-, however, tells us merely that the Ys compose something (maybe not Yorick) that is an intrinsic duplicate of Xavier. No matter. After all, given VIPA+, we can conclude not merely that Yorick has no hand-shaped part, but also that nothing composed of the Ys has a hand-shaped part. Which part would be hand-shaped? Not any of the simples, not any of Yorick's cells (suppose), and not anything composed of all of Yorick's simples. But given VIPA+ and the set-up of the case, these are the only available parts.
} 
Examples of such properties are sometimes taken to include phenomenal properties and certain quantum states possessed by systems of entangled particles (McDaniel 2008). The possibility of strongly emergent properties is apparently incompatible with MS*. Since, according to clause (i), strongly emergent properties must be perfectly natural, and since, in the standard Lewisian framework, all perfectly natural properties are intrinsic, we can conclude that strongly emergent properties must be intrinsic. But clause (iii) tells us, in effect, that things composed of 'matching simples in matching internal arrangements' can differ with respect to these properties. Thus it appears that if strongly emergent properties are possible, then-contrary to $\mathrm{MS}^{*}$ — it is possible for things composed of matching simples in matching arrangements to fail to be intrinsic duplicates. Without MS*, we lose our original motivation to believe that Xavier and Yorick share all their intrinsic properties, and hence we lose our original reason for doubting the Inheritance of Intrinsicality.

Reply. The possibility of strongly emergent properties is in tension with the Intimacy of Parthood itself. Intimacy is the 'picture' according to which a whole is nothing over and above its parts. Sider is concerned to defend Intimacy against the objection that it is incompatible with what he calls 'irreducibly macroscopic features of composite objects' (2007, p. 73). As examples of such properties, he cites quantum states of entangled systems and shapes of composite objects. He claims that such properties are not incompatible with Intimacy, because they

can be pinned on the relations between the parts... For every property $\mathrm{P}$ of a composite object, $\mathrm{x}$, there are some proper parts of $\mathrm{x}$, the Ys, and some relation $\mathrm{R}$, such that the Ys compose $\mathrm{x}$, the Ys stand in $\mathrm{R}, \mathrm{R}$ is at least as natural as $\mathrm{P}$, and necessarily, anything composed of some things standing in $\mathrm{R}$ has P. (2007, pp. 73-74)

If there can be properties that satisfy McDaniel's definition of 'strongly emergent', however, then at least some of them resist being 'pinned on relations' in the way Sider indicates.

To see this, suppose that $\mathrm{P}$ is strongly emergent. Then there can be composite objects $\mathrm{x}$ and $\mathrm{y}$ such that: (i) both $\mathrm{x}$ and $\mathrm{y}$ are composed of simples, (ii) there is a oneto-one correspondence between the simples that compose $\mathrm{x}$ and the simples that compose $y$ that preserves perfectly natural properties and relations, and yet (iii) $\mathrm{x}$ has $\mathrm{P}$ whereas y does not. In that case P cannot be pinned, in Sider's sense, on any equally natural relation that holds amongst x's simple parts. After all, P is strongly emergent, which entails that it is perfectly natural, and in light of the one-to-one correspondence, $x$ 's simples and y's simples are alike in their perfectly natural relations. Now this leaves open the possibility that $\mathrm{P}$ can be pinned on some perfectly natural relation that holds amongst some things that compose $\mathrm{x}$ some of which are nonsimple. But I take it that if strongly emergent properties are possible, then at least some of them could be instantiated by an object all of whose proper parts are simple, e.g., an object composed of just two simples. So if strongly emergent properties are possible, then some of them resist being 'pinned on relations'.

As Sider seems to concede (or at least suggest), such properties would be contrary to the spirit of Intimacy: an object that had such a property would not be 'nothing over and above its parts'. For there would be more to that object's intrinsic 
nature than what is fixed by the intrinsic natures of (and perfectly natural relations between) its proper parts. Granted, this is not exactly an airtight proof that the relevant sorts of properties would run contrary to the Intimacy of Parthood, but (as before) since Intimacy is just a rough picture, we cannot expect much better.

On the assumption that Intimacy is in tension with the possibility of strongly emergent properties, it would be self-defeating for Sider to appeal to that possibility as a way of defusing my objection to the Inheritance of Intrinsicality. We can think of situation as constituting a dilemma for Sider. Either strongly emergent properties are possible or they're not. If they are, then Intimacy becomes highly implausible and cannot function as a premise in a persuasive argument for Uniqueness and UC. If they're not possible, then they do not undermine my argument against the Inheritance of Intrinsicality (i.e., Objection 1 fails). Either way, Sider's case for Uniqueness and UC is in trouble.

Objection 2. So far the argument against the Inheritance of Intrinsicality appears to require VIPA+. If it does, then Sider need not be much bothered by the argument, for it would have extremely limited appeal: VIPA+ has never actually been defended in print.

Reply. The general style of counterexample to the Inheritance of Intrinsicality given here does not depend upon VIPA+. It can be produced in the presence of almost any theory of composition that gives extrinsic factors a role in determining whether composition occurs.

This is a very broad and formidable group of theories. It would seem to include, for example, Commonsensism, the theory that recognizes just simples and the composite objects of scientifically-informed common sense (e.g., DNA molecules, amoebas, artworks, human beings, and planets, but not a fusion of my head and the Eiffel Tower). ${ }^{15}$ I take it that common sense does not recognize any such thing as a fusion of my moles but that it does recognize such things as pieces of body art, and that if I had a twin who was given an exactly similar pattern of duplicate moles (via artificial UV exposure, say) by an artist who then dubbed his work "The Map", this would count as a piece of body art. If so, then Commonsensism tells us that my twin's moles, unlike mine, do have a fusion. ${ }^{16}$ So again we have a counterexample to the Inheritance of Intrinsicality. The precise shape, S, of The Map is intrinsic but having a part that has $S$ is not, for it can differ between duplicates (e.g., my twin and me). This makes it clear, I hope, what a broad range of theories allow for the relevant sort of example.

Those who take such answers seriously constitute a core segment of Sider's target audience. Insofar as Sider is arguing for unrestricted composition, his argument is aimed at those who initially take seriously some other theory of

\footnotetext{
15 Compare this with the view defended in Markosian (1998).

16 The main lesson of Rea (1998) is that if one holds that being arranged artifact-wise (where this is at least partly an extrinsic matter) is sufficient for composing something, then one must either accept unrestricted composition or admit that extrinsic factors are at least sometimes relevant to whether composition occurs. The reason for this is that (plausibly) for any things, no matter what they are like intrinsically and no matter what their internal arrangement, it is possible that things intrinsically just like those, in just that internal arrangement, be arranged artifact-wise (because, say, some artist put them in precisely that arrangement in fulfillment of a very detailed artistic vision).
} 
composition-either nihilism or some moderate theory. As Sider notes, his argument will have very little force on nihilists, since they can explain the truth of the Inheritance principles without appeal to the Intimacy of Parthood. They will (or at least can) explain the truth of those principles by appeal to nihilism itself: given nihilism, the inheritance principles are both vacuously true. Thus it seems that the primary audience for Sider's argument will be those who take moderate theories seriously: composition occurs sometimes but not always.

To be sure, some moderate theories obey an intrinsicness constraint, according to which extrinsic factors are never relevant to whether composition occurs. (van Inwagen takes his own theory to obey such a constraint (1990, pp. 138-140).) Such answers cannot be used to produce Xavier-Yorick-style examples. But these theories tend to conflict with common sense-in addition to conflicting, as all moderate theories do, with the well-known theoretical pressures toward the extreme theories (see Markosian 2008). Most who take moderate theories seriously will do so out of allegiance to common sense, in which case they will also have reason to take seriously those moderate theories (such as Commonsensism and VIPA+) that abandon the intrinsicness constraint. As I see it, these philosophers constitute the bulk of the target audience of Sider's argument for unrestricted composition.

Objection 3. The discussion above may show that much of Sider's target audience has independent reason to doubt the Inheritance of Intrinsicality; this principle is not, as he claims, neutral with regard to theories of composition. But there is another principle in the neighborhood that does have this status. Moreover, this other principle is only slightly weaker than the original and arguably can do the same job as the original in Sider's argument: it can function as a piece of data that is common ground and that is best explained by appeal to the Intimacy of Parthood. The new principle is

Weak Inheritance: If $\mathrm{P}$ is intrinsic, then the property having a part that has $P$ is quasi-intrinsic, i.e., it is a property $\mathrm{G}$ such that necessarily, for any objects $\mathrm{O}$ and $\mathrm{O}^{*}$, if $\mathrm{O}$ and $\mathrm{O}^{*}$ are intrinsic duplicates and 'part-whole isomorphs', then $\mathrm{O}$ has $\mathrm{G}$ iff $\mathrm{O}^{*}$ has $\mathrm{G}^{17}$

To say that $\mathrm{O}$ and $\mathrm{O}^{*}$ are part-whole isomorphs is to say that their parts can be put into a one-to-one correspondence that preserves the part-whole relation, i.e., it is to say that there is a bijective function $\mathrm{f}$ from $\mathrm{O}$ 's parts to $\mathrm{O}^{*}$ 's parts such that for any parts $x$ and $y$ of $O, x$ is a part of $y$ iff $f(x)$ is a part of $f(y)$.

The original Inheritance of Intrinsicality said that if $\mathrm{P}$ is intrinsic, then having a part that has $P$ cannot differ between intrinsic duplicates. Weak Inheritance says merely that if $\mathrm{P}$ is intrinsic, then having a part that has $P$ cannot differ between things that are both intrinsic duplicates and part-whole isomorphs. According to Objection 3, the original principle may admit of counterexamples, but only when the objects involved are not part-whole isomorphs.

\footnotetext{
17 We could also consider a stronger variant, $\mathrm{WI}+$, to the effect that if $\mathrm{P}$ is intrinsic, then having a part that has $\mathrm{P}$ is quasi-intrinsic+, i.e., a property $\mathrm{G}$ such that for any possible worlds $\mathrm{w} 1$ and w2, any object $\mathrm{O} 1$ in w1, and any object $\mathrm{O} 2$ in w2, if $\mathrm{O} 1$ in w1 is an intrinsic duplicate and part-whole isomorph of $\mathrm{O} 2$ in w2, then $\mathrm{O} 1$ has $\mathrm{G}$ in w1 iff $\mathrm{O} 2$ has $\mathrm{G}$ in w2. This would of course be vulnerable to the same objection that I give to Weak Inheritance.
} 
To motivate this thought, suppose that Xavier and Yorick are each composed of exactly five simples. Suppose also that Xavier's parts are his simples, himself, and his right hand (which is itself composed of two of his simples), and that Yorick's parts are his simples and himself. Then Xavier has seven parts, whereas Yorick has just six, in which case their parts cannot even be put into a one-to-one correspondence at all, much less one that preserves the part-whole relation. ${ }^{18}$ Weak Inheritance thus stands unscathed.

Reply. It is easy to construct a Xavier-Yorick-style counterexample to Weak Inheritance that is just as forceful as the original. Let us alter our original example by giving Yorick an artificial right foot to go along with Xavier's artificial right hand, and let us suppose that this foot has the same number of constituent simples as does Xavier's hand. The result will be that the two men are not only intrinsic duplicates (by $\mathrm{MS}^{*}$ ) but also part-whole isomorphs. To see the isomorphism, suppose, as we did above, that each of them is composed of just five simples. Then a one-to-one, part-whole preserving correspondence between their parts can be established as follows:

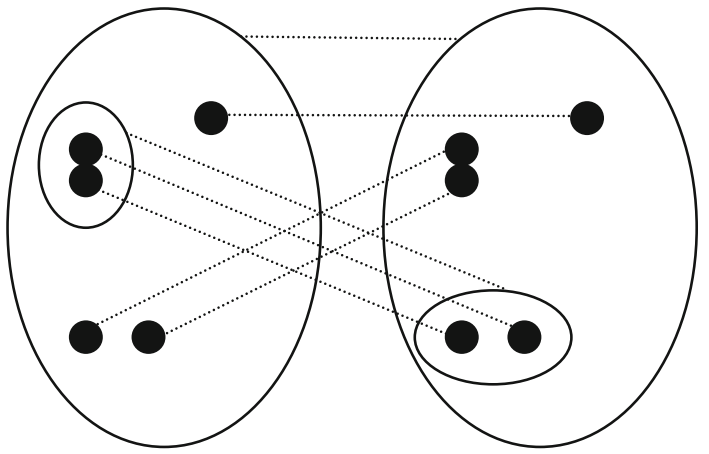

(The large oval on the left represents Xavier; the one on the right represents Yorick; the small oval on the left represents Xavier's hand, the one on the right represents Yorick's foot; the dots represent simples; and being-colocated-with-orinside-of represents being-a-part-of.)

Xavier and Yorick are intrinsic duplicates and part-whole isomorphs, and yet they still differ with respect to the property having a hand-shaped part, despite the

\footnotetext{
18 We could have stipulated instead that each had himself and (say) a countable infinity of simples as parts, and that Xavier, but not Yorick, also had an additional proper part composed of some of his simples. In this case they would have the same number of parts but would still not be part-whole isomorphs, for only Xavier would have a part (any of the simples in his right hand) that is a proper part of two different parts of his (himself and his right hand). To see that this blocks the existence of the relevant sort of correspondence, let $\mathrm{x}$ be such a simple, and let ' $\mathrm{x}<\mathrm{y}$ ' mean ' $\mathrm{x}$ is part of $\mathrm{y}$ '. Then $\mathrm{s}<\mathrm{s}$, $\mathrm{s}<$ Hand, and $\mathrm{s}<$ Xavier, $\mathrm{s} \neq$ Hand, $\mathrm{s} \neq$ Xavier, and Hand $\neq$ Xavier. Now suppose for reductio that there is a one-one, part-whole preserving correspondence f between Xavier's parts and Yorick's. Then Yorick has parts $\mathrm{f}(\mathrm{s}), \mathrm{f}(\mathrm{Hand})$, and $\mathrm{f}($ Xavier $)$. Since $\mathrm{f}$ is one-one, the relevant parts of Yorick must be pairwise distinct (as are the corresponding parts of Xavier), and since $\mathrm{f}$ is part-whole preserving, $\mathrm{f}(\mathrm{s})<\mathrm{f}(\mathrm{s})$, $\mathrm{f}(\mathrm{s})<\mathrm{f}$ (Hand), and $\mathrm{f}(\mathrm{s})<\mathrm{f}($ Xavier $)$. But we know that Yorick does not have such parts: in particular, Yorick has no part such as $\mathrm{f}(\mathrm{s})$ that is a proper part of two different parts of Yorick. Yorick's only parts are himself (which is not a proper part of any of his parts) and his infinitely many simples (each of which his a proper part of Yorick but not of any of Yorick's other parts). So there is no such correspondence.
} 
fact that being hand-shaped is intrinsic. (Now they differ with respect to having a foot-shaped part too.) This violates Weak Inheritance.

Objection 4. The correspondence given above is clearly of the wrong sort, for it preserves the part-whole relation but does not preserve perfectly natural relations between simples. For example, it fails to preserve spatial distance relations: the simples in Xavier's hand are exactly one nanometer apart (suppose), but the 'corresponding' simples in Yorick's foot are not. So what we need is not Weak Inheritance but Very Weak Inheritance:

VWI If $\mathrm{P}$ is intrinsic, then the property having a part that has $P$ is quasi-quasiintrinsic, i.e., it is a property $\mathrm{H}$ such that necessarily, for any objects $\mathrm{O}$ and $\mathrm{O}^{*}$, if $\mathrm{O}$ and $\mathrm{O}^{*}$ are intrinsic duplicates and both composed of simples, ${ }^{19}$ and there is a bijective function $\mathrm{f}$ from the parts of $\mathrm{O}$ to the parts of $\mathrm{O}^{*}$ such that:

(i) for any parts $x$ and $y$ of $O, x$ is part of $y$ iff $f(x)$ is part of $f(y)$,

(ii) for any simple part $\mathrm{x}$ of $\mathrm{O}$ and any perfectly natural property $\mathrm{G}$, $\mathrm{x}$ has $\mathrm{G}$ iff $\mathrm{f}(\mathrm{x})$ has $\mathrm{G}$, and

(iii) for any ordered $\mathrm{n}$-tuple $\left\langle\mathrm{x}_{1}, \ldots, \mathrm{x}_{\mathrm{n}}\right\rangle$ of simple parts of $\mathrm{O}$ and any perfectly natural relation $\mathrm{R}, \mathrm{x}_{1}, \ldots, \mathrm{x}_{\mathrm{n}}$ instantiate $\mathrm{R}$ (in that order) iff $\mathrm{f}\left(\mathrm{x}_{1}\right), \ldots, \mathrm{f}\left(\mathrm{x}_{\mathrm{n}}\right)$ instantiate $\mathrm{R}$ (in that order), then $\mathrm{O}$ has $\mathrm{H}_{\text {iff }} \mathrm{O}^{*}$ has $\mathrm{H}^{20}$

In other words, VWI says that if $\mathrm{P}$ is intrinsic, then having a part that has $P$ cannot differ between intrinsic duplicates (composed of simples) whose parts can be put into a one-to-one correspondence that preserves both the part-whole relation (amongst all the parts) and the perfectly natural properties and relations amongst the simples. This would seem to block all of the counterexamples presented so far.

Reply. I agree that VWI avoids Xavier-Yorick style counterexamples. But this principle is now so much weaker than the original that I doubt it can play the role in the argument that Sider assigned to the original. The original principle asserted an extremely tight link between parts and wholes-to put it aphoristically, that the intrinsic nature of the part is always represented in a certain way within the intrinsic nature of the whole. One might well think that only a picture as bold as the Intimacy of Parthood ("a whole is nothing over and above its parts") offers a fully satisfying explanation of that tight link.

\footnotetext{
19 If this clause were omitted, then the principle would be vulnerable to counterexamples involving gunky intrinsic duplicates, which would satisfy (ii) and (iii) vacuously. Consider the Xavier-Yorick case represented by the diagram above, and alter it in just one way: suppose that each of the dots represents a 'gunky particle' (a particle each of whose parts, itself included, has proper parts). Then it would seem that (a) we could still establish a one-to-one, part-whole preserving correspondence between the parts of Xavier and the parts of Yorick (viz., one that pairs Xavier's hand with Yorick's foot and pairs the particles in the foot with those in the hand), that (b) Xavier and Yorick would still be intrinsic duplicates, and that (c) Xavier and Yorick would still differ with respect to having a hand-shaped part, even though being hand-shaped is intrinsic. Admittedly, in light of Xavier's and Yorick's gunkiness, we couldn't use MS* to argue for (b), but (b) certainly will seem highly plausible to anyone who was attracted to MS*, and there are plausible variants of MS* (arrived at by replacing 'simple' with 'particle') that we could use. So the given clause is crucial.

20 This can be plausibly strengthened in a manner parallel to that suggested for Weak Inheritance, yielding VWI+.
} 
But as soon as we start to doubt the full-strength Inheritance of Intrinsicality and admit that sometimes the intrinsic nature of a part might not be represented in the relevant way within the intrinsic nature of the whole, it is much less clear that we need to appeal to a picture as bold as Intimacy to explain the remaining link between parts and wholes. For more modest explanations become available. VWI, e.g., seems to explicable merely by appeal to MS*, as the latter entails the former. (See the Appendix for a proof.) Of course, MS* might still stand in need of explanation, and it may seem that the Intimacy of Parthood is the best candidate to do the explaining. Can Intimacy earn its keep in this way?

It's not clear that it can. Unlike the Inheritance of Intrinsicality, MS* doesn't say anything about the intrinsic nature of the part always being represented within the intrinsic nature of the whole. It merely says (aphoristically put) that when things are alike at the level of the simples that compose them, then those things will be alike intrinsically 'at the level of the whole'. This is consistent with there being no especially interesting correspondence between the natures of the parts and the natures of the wholes, and it is consistent with there being intrinsic duplicates that are not just alike at the level of the simples that compose them. So, given the great distance between MS* and the Inheritance of Intrinsicality, the burden would be on Sider to motivate the claim that it is Intimacy, rather than something more modest, that provides the best explanation of MS*.

Objection 5. The Inheritance of Intrinsicality tells us that the nature of the part is represented within the nature of whole-as Sider puts it, that 'the part shines through' (2007, p. 70). VWI tells us no such thing; it allows the part to be 'blotted out'. But Sider need not retreat to anything as weak as VWI. Instead he can conjoin VWI with:

Simple Inheritance (a) If property $\mathrm{P}$ is intrinsic, then the property having a simple part that has $P$ is intrinsic; and (b) if relation $\mathrm{R}$ is perfectly natural, then the property having some simple parts that instantiate $R$ is intrinsic. ${ }^{21}$

What sorts of composite parts a thing has might be an extrinsic affair, if some externalist theory of composition is true. Even so, what sorts of simple parts a thing has (and how they are related) would plausibly remain intrinsic. So Simple Inheritance is not threatened by the examples so far considered. Moreover, while Simple Inheritance does not go so far as to say that all parts 'shine though' in the relevant sense, it does say that at least the simple parts do. This goes well beyond MS* or VWI and calls for a correspondingly stronger explanation.

Granted, Simple Inheritance does leave open the possibility of objects none of whose proper parts 'shine through', for there might still be gunky objects. (An object is gunky just in case each of its parts, itself included, has proper parts; a gunky object has no simple parts.) But Sider can appeal to additional principles to cover those cases:

G1 The property being gunky is intrinsic.

$\overline{21}$ Thanks to Ted Sider for suggesting something in this neighborhood. 
G2 The property having a gunky part is intrinsic.

The first ensures that if an object is gunky, this fact is reflected in its intrinsic nature. Together, the first and the second guarantee that even gunky parts 'shine through,' at least in a limited way. Whereas Simple Inheritance ensures that the complete intrinsic nature of a simple part of a thing will be reflected in the intrinsic nature of the whole, G1 and G2 ensure that at least one aspect of the intrinsic nature of a gunky part of a thing (the part's gunkiness) is reflected within the intrinsic nature of the whole. So once again, Sider has uncontroversial data (the conjunction of VWI, Simple Inheritance, G1, and G2) that is best explained by appeal to the Intimacy of Parthood.

Reply. This is a definite improvement over the two previous suggestions. But there is still a significant gap between the new 'conjunctive principle' and the original Inheritance of Intrinsicality. The conjunctive principle guarantees only that the gunkiness of a gunky part is reflected in the intrinsic nature of the whole. According to the Inheritance of Intrinsicality, if a thing $A$ has a part $b$, then, regardless of whether $b$ is gunky, every intrinsic property of $b$ is reflected in A's intrinsic nature: e.g., if having a mass of 1 unit is an intrinsic property of $b$, then having a part with mass of 1 unit is an intrinsic property of A.

We give this up when we shift to the conjunctive principle. It tells us that if $b$ is simple, then all of its intrinsic properties are reflected in A's nature, and if $b$ is composite but composed of simples, then at least the complete natures of its simples (and their arrangement) will be reflected in A's nature. But if $b$ is gunky, then, for all the conjunctive principle tells us, much of $b$ 's nature may be 'blotted out' and go completely unrepresented in A's nature: e.g., even if having a mass of 1 unit is an intrinsic property of $b$, having a part that has a mass of 1 unit might (for reasons stemming from Xavier-Yorick-style cases, e.g.) merely be an extrinsic property of A. And if A itself is gunky, then what is true of $b$ may be true of all of A's proper parts, so that A's intrinsic nature reflects relatively little about the intrinsic natures of any of its proper parts.

So there is a significant gap between the Inheritance of Intrinsicality and the Conjunctive Principle. As a result, even if the Intimacy of Parthood would be needed to explain the former principle, there is still plenty of room to doubt that Intimacy is needed to explain the latter. In light of the distance between the original data and the new data, Sider's case for saying that Intimacy is the best explanation of the old data does not transfer directly to the new data. ${ }^{22}$

\footnotetext{
22 A referee suggested replacing Simple Inheritance with a certain principle that he or she suggested might be equally plausible but stronger and more robust as data in support of Intimacy: roughly, if $\mathrm{P}$ is intrinsic, then the property having a part that has $P$ and that does not 'owe its existence to outside factors' is intrinsic. Since there are, presumably, composite objects and even gunky objects that do not owe their existence to outside factors (in the manner of Xavier's hand), this principle, unlike Simple Inheritance, would have the consequence that the properties of such composite objects do 'shine through'. Whether this move helps depends on whether we can make sense of the notion of owing one's existence to outside factors. On this latter question I am agnostic. Although I have made several attempts to define the notion without success (and without achieving any insights that would justify discussing these attempts in print, which would be somewhat lengthy and tedious), I admit that others might do better, and that, alternatively, the notion might be a legitimate primitive.
} 
Objection 6. What Xavier-Yorick-style examples show is that a certain set S of propositions is inconsistent. S includes: (i) the Inheritance of Intrinsicality, (ii) MS*, (iii) VIPA+ (or some relevantly similar theory of composition, such as Commonsensism), and (iv) some proposition to the effect that simples are arranged in the manner indicated in the example and have the indicated histories. This does not cast doubt on the Inheritance of Intrinsicality; rather, it casts doubt on the conjunction of MS* and VIPA+.

Reply. Different philosophers will of course respond to the example in different ways. I have no intention of arguing that (ii) and (iii) are so plausible that everyone ought to respond by doubting (i). But I do think that they are sufficiently plausible, or at least taken seriously by a sufficiently large group of metaphysicians, that the inconsistency of set $\mathrm{S}$ is significant. Whether this inconsistency is used to argue against (i), (ii), or (iii), the resulting argument will be of interest.

For example, there has been intense controversy surrounding microphysical supervenience principles in the neighborhood of MS*. ${ }^{23}$ The fact that MS* conflicts with the conjunction of (i), (iii) and (iv) will give some philosophers a new reason to doubt it.

Likewise, I suspect that many philosophers have felt vaguely uncomfortable with externalist theories of composition like VIPA+ and Commonsensism and yet have been unable to formulate a non-question-begging argument against those theories. This seems to be Peter van Inwagen's predicament. Concerning VIPA+ (or a close variant of it), he writes that

This answer goes against all my deepest instincts... [A] proper answer to the Special Composition Question must conform to the following principle:

If the xs compose something, and if the ys perfectly duplicate the xs (both in their intrinsic properties and in the spatiotemporal and causal relations they bear to one another), then the ys compose something.

... I do not know how to defend my instinctive allegiance to this principle except by trying, as I have tried, to present the principle in as attractive a light as possible. (1990, p. 140)

The argument from (i), (ii), and (iv) to the negation of (iii) strikes me as being just the sort of thing that van Inwagen is looking for. ${ }^{24}$

And, just as a matter of sociology, I predict that some philosophers will respond to the example in just the way I've been highlighting here-by reducing their credence in (i).

Objection 7. The case against MS* is more powerful than you acknowledge. After all, the following argument against MS*, which the VIPA+-lover is in a position to offer, does not rely on the Inheritance of Intrinsicality:

\footnotetext{
23 See, e.g., Merricks (1998a, b), Hawley (1998), Noonan (1999a, b).

24 Cameron (2007) responds to a related argument that I suggested in my commentary on that paper at the 2006 Bellingham Summer Philosophy Conference.
} 
Xavier and Yorick each have, say, exactly 1,000 simple parts. Given VIPA+, Xavier has two additional (non-simple) parts, his hand and himself; and Yorick, by contrast, has only one non-simple part, namely himself. So Xavier has 1,002 parts overall, whereas Yorick has only 1,001 parts overall. Together with the principle that

INP for any number $\mathrm{n}$, the property having exactly $n$ parts is intrinsic,

this entails that Xavier and Yorick are not intrinsic duplicates, contrary to MS*.

So it's not just the Inheritance of Intrinsicality that would be threatened by conjunction of MS* and VIPA+; INP would be too. This reflects badly on MS*.

Reply. Two points are relevant here. First, rejecting INP is not much costlier than rejecting the Inheritance of Intrinsicality. I take it that upon initially encountering the case of Xavier and Yorick, virtually everyone will find it natural to assume that (given VIPA+) Xavier has one more part than Yorick. If, in the context of that assumption, one is willing to take the case seriously as a counterexample to the Inheritance of Intrinsicality, then why shouldn't one take it seriously as a counterexample to INP as well?

Second, remember that we have the option of putting the blame on VIPA+ (or whatever externalist theory of composition we're using) rather than on MS*. For what it's worth, if we set aside the possibility of strongly emergent properties and relations, I would much sooner reject VIPA+ than MS*, and I'm not alone in this. But I confess that I have no argument for this preference beyond an appeal to intuition.

Objection 8. Perhaps there are different senses of 'intrinsic', some of which make the Inheritance of Intrinsicality true and others of which don't, but all of which are legitimate, reasonably natural sharpenings of the term as it has been used in the recent literature. In that case, couldn't Sider just stipulate that for the purposes of his argument, only the former senses of 'intrinsic' are relevant?

Reply. To admit that the Inheritance of Intrinsicality will fail (or at least be controversial) on some legitimate, reasonably natural sharpenings of 'intrinsic' would be to diminish the force of the argument. If that principle were uncontroversial on all legitimate sharpenings of 'intrinsic', this would be a much more robust piece of data, and it would be much more likely to support Intimacy, than if the Inheritance principle were merely uncontroversial on some legitimate sharpenings while being controversial on others. I take it that Sider's claim is that the Inheritance principle is uncontroversial on all such sharpenings; see, e.g., (2007, p. 75).

\section{Conclusion}

Classical mereology is opposed by two partially overlapping groups of dissenters: coincidentalists, who embrace coinciding objects and deny Uniqueness, and composition restricters, who deny UC and say that some pluralities of objects lack fusions. How do these groups fare against Sider's counteroffensive? 
Some will find the Intimacy of Parthood so nebulous as to be incapable of explaining any data, or of yielding any predictions. Others will be more concessive. They will allow that Intimacy is intelligible, that it is supported by the data to which Sider appeals, and that it supports UC and Uniqueness. But in the end they will reject it anyway, simply because they take the case against UC and/or Uniqueness to outweigh the case for Intimacy.

I have explored a pair of responses that engage with Sider's arguments in a more substantive way. On behalf of the coincidentalists, I have suggested that they can concede the case for Intimacy while resisting the step from Intimacy to Uniqueness. On behalf of composition restricters, I have suggested that some of them-those who are firmly attached to the relevant doctrines concerning microphysical supervenience and externalism about composition-will have independent reason to reject some of the data that Sider uses to support Intimacy. But even those who are not so firmly attached to microphysical supervenience and externalism about composition may have something to learn from the Xavier-Yorick case, for they can see it as providing a new and unanticipated reason to reject the conjunction of the given doctrines.

Acknowledgments Thanks to Kris McDaniel, Adam Sennet, Alexander Skiles, Ted Sider and a referee for helpful comments.

Open Access This article is distributed under the terms of the Creative Commons Attribution Noncommercial License which permits any noncommercial use, distribution, and reproduction in any medium, provided the original author(s) and source are credited.

\section{Appendix: Proof that MS* entails VWI}

Suppose that (a) o and $o^{*}$ are intrinsic duplicates, that (b) they're both composed of simples, and that (c) their parts can be put into a one-one correspondence $f$ that preserves both the part-whole relation (amongst all parts) and perfectly natural properties and relations (amongst their simple parts). Finally, suppose that (d) P is an intrinsic property.

To show that MS* entails VWI, it suffices to show that (a)-(d) plus MS* entails that o has the property having a part that has $P$ iff $\mathrm{o}^{*}$ does. Since our assumptions do not distinguish o from $\mathrm{o}^{*}$, we can prove the biconditional by proving just one direction of it. So suppose that o has the property having a part that has $P$. Then there is a part of o, call it $o-$, that has P. Since o is composed of some simples (call them the Os) and o- is a part of $\mathrm{o}$, we can conclude that $\mathrm{o}-$ is also composed of some simples (the $\mathrm{O}-\mathrm{s}$ ) and that the $\mathrm{O}-\mathrm{s}$ are among the Os: the set of the former is a (proper or improper) subset of the set of the latter. Since $f$ is part-whole preserving and hence pairs simples only with simples, it will give us some simple parts of $\mathrm{o}^{*}$, call them the $\mathrm{O}^{*}-\mathrm{s}$, that correspond to the $\mathrm{O}-\mathrm{s}$. Moreover, the fact that $\mathrm{f}$ is part-whole preserving also entails that the $\mathrm{O} *-\mathrm{s}$ compose $\mathrm{f}(\mathrm{o}-)$. Since $\mathrm{f}$ preserves perfectly natural properties and relations between the simple parts of o (the Os) and the simple parts of $\mathrm{o}^{*}$ (the $\mathrm{O}^{*} \mathrm{~s}$ ), and since the $\mathrm{O}^{*}-\mathrm{s}$ are among the $\mathrm{O}^{*}$ s just as the $\mathrm{O}-\mathrm{s}$ are among the $\mathrm{Os}$, f also preserves perfectly natural properties and relations between the $\mathrm{O}-\mathrm{s}$ and the $\mathrm{O}^{*}-\mathrm{s}$. 
So we now know this: (i) $\mathrm{o}-$ is composed of the $\mathrm{O}-\mathrm{s}$, (ii) the $\mathrm{O}-\mathrm{s}$ are all simples, (iii) $\mathrm{f}(\mathrm{o}-)$ is composed of the $\mathrm{O}^{*}-\mathrm{s}$, (iv) the $\mathrm{O}^{*}-\mathrm{s}$ are all simples, (v) there is a one-one correspondence between the $\mathrm{O}-\mathrm{s}$ and the $\mathrm{O} *-\mathrm{s}$ that preserves perfectly natural properties and relations, (vi) $\mathrm{P}$ is intrinsic, and (vii) $\mathrm{O}-$ has $\mathrm{P}$. Conjoined with MS*, this entails that $\mathrm{f}(\mathrm{o}-)$ has $\mathrm{P}$ too. ${ }^{25}$ Since $\mathrm{f}(\mathrm{o}-)$ is a part of $\mathrm{o}^{*}$, it follows that $\mathrm{O}^{*}$ has a part that has $\mathrm{P}$ and hence that $\mathrm{O}^{*}$ instantiates the property having a part that has $P$. So MS* entails VWI. ${ }^{26}$

\section{References}

Cameron, R. (2007). The contingency of composition. Philosophical Studies, 136, 99-121.

Hawley, K. (1998). Merricks on whether consciousness is intrinsic. Mind, 107, 841-843.

Lewis, D. (1986). On the plurality of worlds. London: Blackwell.

Markosian, N. (1998). Brutal composition. Philosophical Studies, 92, 211-249.

Markosian, N. (2008). Restricted composition. In T. Sider, J. Hawthorne, \& D. W. Zimmerman (Eds.), Contemporary debates in metaphysics (pp. 341-363). London: Blackwell.

McDaniel, K. (2003). No paradox of multi-location. Analysis, 63, 309-311.

McDaniel, K. (2008). Against composition as identity. Analysis, 68, 128-133.

Merricks, T. (1998a). Against the doctrine of microphysical supervenience. Mind, 107, 59-72.

Merricks, T. (1998b). On whether being conscious is intrinsic. Mind, 107, 845-846.

Noonan, H. (1999a). Microphysical supervenience and consciousness. Mind, 108, 755-759.

Noonan, H. (1999b). Identity, constitution and microphysical supervenience. Proceedings of the Aristotelian Society, 99(3), 273-288.

Rea, M. (1998). In defense of mereological universalism. Philosophy and Phenomenological Research, 58, 347-360.

Sider, T. (2007). Parthood. The Philosophical Review, 116, 51-91.

Skow, B. (2007). Are shapes intrinsic? Philosophical Studies, 113, 111-130.

Van Inwagen, P. (1990). Material beings. Ithaca: Cornell.

\footnotetext{
${ }^{25} \mathrm{MS}^{*}$ - does not have this effect; it merely guarantees that the $\mathrm{O}^{*}-\mathrm{s}$ compose something (call it $q$ ) that has $\mathrm{P}$, not that $\mathrm{f}(\mathrm{o}-)$ itself has $\mathrm{P}$. If $\mathrm{q}$ (unlike $\mathrm{f}(\mathrm{o}-)$ ) might fail to be a part of $\mathrm{o}-$, then we have no guarantee that $\mathrm{o}-$ has a part that has $\mathrm{P}$. We do get this guarantee, however, if we adopt the popular (though not universally accepted) mereological principle strong supplementation: if every part of $\mathrm{x}$ overlaps $y$, then $x$ is a part of $y$. Since every part of $\mathrm{p}$ is either a simple part of $\mathrm{o}-$ or is entirely composed of simple parts of $\mathrm{o}-$, it follows that every part of $\mathrm{q}$ overlaps $\mathrm{o}-$, which together with strong supplementation entails that $\mathrm{q}$ itself is a part of $\mathrm{o}-$.

${ }^{26}$ Likewise, VWI+ is entailed by MS*+. The proof is parallel.
} 